\title{
STRONG ASYMPTOTICS FOR JACOBI POLYNOMIALS WITH VARYING WEIGHTS
}

\section{Christof Bosbach and Wolfgang Gawronski}

\section{Dedicated to Richard Askey on the occasion of his 65th birthday}

\begin{abstract}
Generalizing classical and recent results for Jacobi polynomials $P_{n}^{\left(\alpha_{n}, \beta_{n}\right)}(z)$, strong asymptotics are established as $n \rightarrow \infty$ where, in contrast to the standard situation, the real parameters $\alpha_{n}, \beta_{n}$ depend on the degree $n$ and satisfy certain limiting conditions. By rescaling the argument $z$ suitably, extensions of the well-known Darboux formulae are derived.
\end{abstract}

\section{Introduction and summary}

In this paper, we deal with generalizations of asymptotic forms for Jacobi polynomials $P_{n}^{(\alpha, \beta)}$. As usual, we assume these functions to be defined via the orthogonality relation

$$
\begin{aligned}
\int_{-1}^{1} & P_{n}^{(\alpha, \beta)}(x) P_{m}^{(\alpha, \beta)}(x)(1-x)^{\alpha}(1+x)^{\beta} d x \\
& =\frac{2^{\alpha+\beta+1}}{2 n+\alpha+\beta+1} \frac{\Gamma(n+\alpha+1) \Gamma(n+\beta+1)}{n ! \Gamma(n+\alpha+\beta+1)} \delta_{n m}, \quad \alpha, \beta>-1,
\end{aligned}
$$

which we take from Szegö's book [25, Chapter IV] together with the normalization and some standard formulae as well.

The classical asymptotic theory of $P_{n}^{(\alpha, \beta)}$ is concerned with large degrees $n$ and fixed parameters $\alpha, \beta$ [25, Chapter VIII]. We mention Darboux's formulae [25, p. 196]

$$
P_{n}^{(\alpha, \beta)}(z)=\frac{1}{\sqrt{\pi n}} \frac{w^{n+1}}{\left(w^{2}-1\right)^{1 / 2}}\left(\frac{2 w}{w-1}\right)^{\alpha}\left(\frac{2 w}{w+1}\right)^{\beta}(1+o(1))
$$

for $z \in \mathbb{C}_{[-1,1]}$ and

$$
\begin{aligned}
P_{n}^{(\alpha, \beta)}(\cos \theta)= & \frac{1}{\sqrt{\pi n}}\left(\sin \frac{\theta}{2}\right)^{-\alpha-\frac{1}{2}}\left(\cos \frac{\theta}{2}\right)^{-\beta-\frac{1}{2}} \\
& \times\left\{\sin \left(\left(n+\frac{\alpha+\beta+1}{2}\right) \theta-\left(\alpha+\frac{1}{2}\right) \frac{\pi}{2}\right)+O\left(\frac{1}{n}\right)\right\}
\end{aligned}
$$

for $\theta \in(0, \pi)$ as $n \rightarrow \infty$. Here and throughout, for real $r, s$ with $r<s$, we use the notation

$$
\mathbb{C}_{[r, s]}:=\mathbb{C} \backslash[r, s]
$$

Received November 4, 1997, revised May 23, 1998.

1991 Mathematics Subject Classification: 30E15, 33C45.

Key words and phrases: Jacobi polynomials, strong asymptotics, Darboux formulae. 
i.e., the complex plane with a cut along the interval $[r, s]$, and Jukowski's function

$$
w:=\frac{\sqrt{z+1}+\sqrt{z-1}}{\sqrt{z+1}-\sqrt{z-1}}=z+\sqrt{z^{2}-1}
$$

mapping $\mathbb{C}_{[-1,1]}$ conformally onto the exterior of the unit circle $|w|>1$ such that $z=\infty$ corresponds to $w=\infty$. Both asymptotic forms (1.2), (1.3) hold uniformly on compact subsets of $\mathbb{C}_{[-1,1]}$ and of $(-1,1)$, the interval of zeros, respectively. It is customary to denote this property by the phrase strong asymptotics, in contrast to the so-called weak asymptotics, that is the limit distribution for the zeros. We shall use this terminology throughout for the Jacobi polynomials $P_{n}^{\left(\alpha_{n}, \beta_{n}\right)}$ in this paper as well.

Questions of weighted polynomial approximation led Lachance, Moak, Saff, Ullman and Varga $[12,18,23]$ to investigate the modified Jacobi polynomials $P_{n}^{\left(\alpha_{n}, \beta_{n}\right)}$ where the real parameters $\alpha_{n}, \beta_{n}$ may depend on $n$. If both $\alpha_{n}, \beta_{n}>-1$, then it is well known that the zeros of $P_{n}^{\left(\alpha_{n}, \beta_{n}\right)}, x_{n \nu}, \nu=1, \ldots, n$, say, are located in the open interval $(-1,1)$, and that all of them are simple. In addition, suppose that

$$
A_{n}:=\frac{\alpha_{n}}{2 n+\alpha_{n}+\beta_{n}}, \quad B_{n}:=\frac{\beta_{n}}{2 n+\alpha_{n}+\beta_{n}},
$$

and that the limits

$$
A:=\lim _{n \rightarrow \infty} A_{n}, \quad B:=\lim _{n \rightarrow \infty} B_{n}
$$

both exist. Then, by using Sturm's comparison theory in [18], Moak et al. proved that the set

$$
\bigcup_{n=1}^{\infty}\left\{x_{n 1}, \ldots, x_{n n}\right\}
$$

of zeros is dense in the interval $[r, s]$ where

$$
r:=B^{2}-A^{2}-\sqrt{D}, \quad s:=B^{2}-A^{2}+\sqrt{D},
$$

and

$$
D:=(1-A+B)(1+A-B)(1-A-B)(1+A+B) .
$$

Note that (1.6)-(1.8) imply $0 \leq A, B, A+B, D \leq 1$ and $[r, s] \subset[-1,1]$. Sharpening the latter result in a series of papers $[4-6,9,10,16,17,22,23]$, weak asymptotics for $P_{n}^{\left(\alpha_{n}, \beta_{n}\right)}$ have been established, as well as in a more general frame for orthogonal polynomials with varying weights $w_{n}(x)$ satisfying $\lim _{n \rightarrow \infty} w_{n}(x)^{1 / n}=w(x)[9,10$, $13-15,21,24,26,28]$. The recent publications $[4,5]$, among others, treat weak asymptotics for $P_{n}^{\left(\alpha_{n}, \beta_{n}\right)}$ under the general assumptions $\alpha_{n}, \beta_{n}>-1$ and

$$
\lim _{n \rightarrow \infty} \frac{\alpha_{n}}{n}=a, \quad \lim _{n \rightarrow \infty} \frac{\beta_{n}}{n}=b
$$

where, in particular, the cases $a=\infty$ and/or $b=\infty$ are admissible.

It is the main purpose of this paper to derive strong asymptotics for $P_{n}^{\left(\alpha_{n}, \beta_{n}\right)}$ under the general condition (1.9). For the very special case of linear parameter sequences $\alpha_{n}=a n+\alpha, \beta_{n}=b n+\beta$, with $a, b \geq 0$ and $\alpha, \beta \in \mathbb{R}$ being independent of $n$, this has been done in $[3,8]$. Besides (1.5), we put

$$
D_{n}:=\left(1-A_{n}+B_{n}\right)\left(1+A_{n}-B_{n}\right)\left(1-A_{n}-B_{n}\right)\left(1+A_{n}+B_{n}\right),
$$


and we distinguish two cases. If both limits $a, b$ in (1.9) are finite, that is $A+B<1$ in (1.6), then we consider the polynomials $P_{n}^{\left(\alpha_{n}, \beta_{n}\right)}(z)$, whereas in case $a=\infty$ or $b=\infty$ in (1.9), that is $A+B=1$ in (1.6), we consider the rescaled polynomial $P_{n}^{\left(\alpha_{n}, \beta_{n}\right)}\left(\sqrt{D_{n}} z+B_{n}-A_{n}\right)$. This particular scaling of the argument is made in light of the weak asymptotics treated in $[4$, p. $231 ; 5$, p. 244]. The resulting generalizations and extensions of Darboux's formulae in (1.2), (1.3) are derived in Sections 3 and 4.

The proofs of the various asymptotic forms rely on the saddle point approximation of certain contour integrals combined with Vitali's theorem on compact convergence for sequences of analytic functions. Also we mention that strong asymptotics for Laguerre polynomials $L_{n}^{\left(\alpha_{n}\right)}$ with varying weights are proved in [2].

\section{Preliminary results}

In this section, we collect some auxiliary results and formulae which are fundamental for the proofs of our main theorems.

We start with the well-known representation

$$
P_{n}^{(\alpha, \beta)}(z)=\frac{1}{2 \pi i} \int_{C}\left(\frac{1}{2} \frac{t^{2}-1}{t-z}\right)^{n}\left(\frac{1-t}{1-z}\right)^{\alpha}\left(\frac{1+t}{1+z}\right)^{\beta} \frac{d t}{t-z}, \quad z \neq \pm 1,
$$

by means of a contour integral [25, p. 70, (4.4.6)], $\alpha, \beta \in \mathbb{C}, n \in \mathbb{N}_{0}$. Here $C$ is a simple closed contour with positive orientation enclosing the point $t=z$ but not the points $t= \pm 1$. The fractional powers of $(1-t) /(1-z)$ and $(1+t) /(1+z)$ are assumed to reduce to 1 for $t=z$.

For the real sequences $\left(\alpha_{n}\right),\left(\beta_{n}\right)$ with $\alpha_{n}, \beta_{n}>-1$, we suppose that (1.9) is satisfied. Obviously, we have $a, b \in[0, \infty]$ and, on account of $(1.5),(1.6)$, that $a=\infty$ or $b=\infty$ occur if and only if $A+B=1$. Thus, in view of the above mentioned weak asymptotics, we consider the modified Jacobi polynomials $P_{n}^{\left(\alpha_{n}, \beta_{n}\right)}(z)$ when both $a, b$ are finite, and if $a=\infty$ or $b=\infty$, then we deal with the rescaled Jacobi polynomials $P_{n}^{\left(\alpha_{n}, \beta_{n}\right)}\left(\sqrt{D_{n}} z+B_{n}-A_{n}\right)$ where $D_{n}$ is given in (1.10). Now (2.1) can be written as

$$
P_{n}^{\left(\alpha_{n}, \beta_{n}\right)}(z)=\frac{1}{2 \pi i} \int_{C} e^{-q_{n}(t)} d t, \quad z \neq \pm 1
$$

if $a, b \in[0, \infty)$ where

$$
q_{n}(t):=n \log \frac{2(t-z)}{t^{2}-1}+\alpha_{n} \log \frac{1-z}{1-t}+\beta_{n} \log \frac{1+z}{1+t}+\log (t-z) .
$$

If $a=\infty$ or $b=\infty$, then from (2.1), we infer

$$
\begin{gathered}
P_{n}^{\left(\alpha_{n}, \beta_{n}\right)}\left(\sqrt{D_{n}} z+B_{n}-A_{n}\right)=\frac{1}{2 \pi i D_{n}^{n / 2}} \int_{C_{n}} e^{-p_{n}(t)} d t \\
z \neq\left( \pm 1+A_{n}-B_{n}\right) / \sqrt{D_{n}}, \text { with } \\
p_{n}(t):=n \log \left(\frac{2(t-z)}{\left(B_{n}-A_{n}+\sqrt{D_{n}} t\right)^{2}-1}\right)+\log (t-z) \\
+\alpha_{n} \log \left(\frac{1-B_{n}+A_{n}-\sqrt{D_{n}} z}{1-B_{n}+A_{n}-\sqrt{D_{n}} t}\right)+\beta_{n} \log \left(\frac{1+B_{n}-A_{n}+\sqrt{D_{n}} z}{1+B_{n}-A_{n}+\sqrt{D_{n}} t}\right)
\end{gathered}
$$

and $C_{n}$ being a simple closed contour with positive orientation enclosing $t=z$ but not the points $t=\left( \pm 1+A_{n}-B_{n}\right) / \sqrt{D_{n}}$. Further, according to the choice of the fractional powers in (2.1) for the logarithms in (2.3) and (2.5) we have to choose that branch for 
which $\log \xi$ is real, when $\xi$ is real and positive. In the sequel, an asymptotic evaluation of the modified Jacobi polynomials is performed by a saddle point approximation of the contour integrals in (2.2) and (2.4). The special case of linear sequences, that is $\alpha_{n}=a n+\alpha, \beta_{n}=b n+\beta, a, b \geq 0$, in (2.2) [8, p. 384], could be dealt with by the saddle point method in standard form [11, 20,27,31]. A treatment of the general cases in (2.2) and (2.4) requires a variant of the saddle point approximation which we take from [1, Section 20.2]. It is given by the following lemma (also compare with the corresponding Laguerre case [2]).

Lemma 2.1. Suppose that $G \subset \mathbb{C}$ is a domain and $g_{n}$ are complex-valued functions, $n \in \mathbb{N}$, being twice differentiable on $G$. Further, let $t_{n} \in G, \omega_{n}$ be complex numbers such that the straight lines $T_{n}=\left[t_{n}-\omega_{n}, t_{n}+\omega_{n}\right]$ are contained in $G, n \in \mathbb{N}$. If $(\epsilon>0)$

$$
\begin{gathered}
g_{n}^{\prime}\left(t_{n}\right)=0, \quad n \in \mathbb{N}, \\
\lim _{n \rightarrow \infty}\left|\omega_{n}^{2} g_{n}^{\prime \prime}\left(t_{n}\right)\right|=\infty, \\
\left|\arg \left(\omega_{n} g_{n}^{\prime \prime}\left(t_{n}\right)^{1 / 2}\right)\right| \leq \frac{\pi}{4}-\epsilon, \quad n \geq n_{0}(\epsilon), \\
g_{n}^{\prime \prime}(t)=g_{n}^{\prime \prime}\left(t_{n}\right)(1+o(1)) \quad \text { as } n \rightarrow \infty, \\
\text { uniformly with respect to } t \in T_{n},
\end{gathered}
$$

then we have

$$
\int_{T_{n}} e^{-g_{n}(t)} d t=\sqrt{\frac{2 \pi}{g_{n}^{\prime \prime}\left(t_{n}\right)}} e^{-g_{n}\left(t_{n}\right)}(1+o(1)), \quad n \rightarrow \infty .
$$

Condition (2.8) is to fix the correct branch of the square root $g_{n}^{\prime \prime}\left(t_{n}\right)^{1 / 2}$.

Further, we need the following Poisson integrals which are important for transferring asymptotics of $P_{n}^{\left(\alpha_{n}, \beta_{n}\right)}(x), x$ being real, to $P_{n}^{\left(\alpha_{n}, \beta_{n}\right)}(z), z$ being complex [25, pp. 275$277 ; 7$, p. $40 ; 2]$.

Lemma 2.2. Suppose that the real numbers $c$, $d$ satisfy $c>0, c \geq|d|$ and $\zeta$ is a complex number with $|\zeta|<1$. Then

$$
\frac{1}{2 \pi} \int_{-\pi}^{\pi} \log \left(c^{2}+d^{2}+2 c d \cos \theta\right) \frac{1+\zeta e^{-i \theta}}{1-\zeta e^{-i \theta}} d \theta=2 \log (c+d \zeta),
$$

$\log$ being the principal branch (i.e., $\log x$ is real if $x$ is real and positive), and

$$
\frac{1}{2 \pi} \int_{-\pi}^{\pi} \log \left(\sin ^{2} \theta\right) \frac{1+\zeta e^{-i \theta}}{1-\zeta e^{-i \theta}} d \theta=\log \left\{\left(\frac{\zeta}{2}\left(\zeta-\frac{1}{\zeta}\right)\right)^{2}\right\} .
$$

3. The case $\alpha_{n} / n \rightarrow \infty$ or $\beta_{n} / n \rightarrow \infty$

In this section, we suppose that at least one of the quantities $a, b$ in (1.9) is infinite and, in addition, that $\alpha_{n}, \beta_{n} \geq 1$ for $n \in \mathbb{N}$. Strong asymptotics for the modified Jacobi polynomials $P_{n}^{\left(\alpha_{n}, \beta_{n}\right)}\left(\sqrt{D_{n}} z+B_{n}-A_{n}\right)$ now is derived via saddle point approximation of the parameter integral in (2.4). Therefore, basically, we follow ideas used in $[2,7$, 8].

The saddle point equation $p_{n}^{\prime}(t)=0$ for the integral in (2.4) takes the form

$$
\frac{n+1}{t-z}-\frac{\alpha_{n}+n}{t-\left(1-B_{n}+A_{n}\right) / \sqrt{D_{n}}}-\frac{\beta_{n}+n}{t+\left(1+B_{n}-A_{n}\right) / \sqrt{D_{n}}}=0
$$


with the explicit solutions

$$
t_{n}^{ \pm}(z)=\frac{2 n+\alpha_{n}+\beta_{n}}{2\left(n+\alpha_{n}+\beta_{n}-1\right)}\left(z-\frac{r_{n}+s_{n}}{2} \pm \sqrt{\left(z-r_{n}\right)\left(z-s_{n}\right)}\right)
$$

where

$$
\begin{aligned}
& r_{n}=\frac{\left(\alpha_{n}-\beta_{n}\right)(n+1)}{2 \sqrt{n\left(n+\alpha_{n}\right)\left(n+\beta_{n}\right)\left(n+\alpha_{n}+\beta_{n}\right)}}-\sqrt{\frac{(n+1)\left(\alpha_{n}+\beta_{n}+n-1\right)}{n\left(\alpha_{n}+\beta_{n}+n\right)}}, \\
& s_{n}=\frac{\left(\alpha_{n}-\beta_{n}\right)(n+1)}{2 \sqrt{n\left(n+\alpha_{n}\right)\left(n+\beta_{n}\right)\left(n+\alpha_{n}+\beta_{n}\right)}}+\sqrt{\frac{(n+1)\left(\alpha_{n}+\beta_{n}+n-1\right)}{n\left(\alpha_{n}+\beta_{n}+n\right)}} .
\end{aligned}
$$

In view of (1.9), obviously we have $r_{n} \rightarrow r, s_{n} \rightarrow s$ as $n \rightarrow \infty$ where

$$
\begin{gathered}
r=-1+\frac{1}{2 \sqrt{1+b}}, \quad s=1+\frac{1}{2 \sqrt{1+b}}, \quad \text { if } a=\infty, b \in[0, \infty), \\
r=-1-\frac{1}{2 \sqrt{1+a}}, \quad s=1-\frac{1}{2 \sqrt{1+a}}, \quad \text { if } a \in[0, \infty), b=\infty, \\
r=-1, \quad s=1, \quad \text { if } a=b=\infty
\end{gathered}
$$

(compare Theorem 2, ii) in [5]). In order to determine the proper branch of the square root function in (3.2) for $z \in \mathbb{C}_{\left[r_{n}, s_{n}\right]}$, we introduce the variable $w_{n}$ by

$$
\begin{aligned}
z= & \frac{r_{n}+s_{n}}{2}+\frac{s_{n}-r_{n}}{2} \frac{w_{n}+w_{n}^{-1}}{2} \\
= & \frac{\left(\alpha_{n}-\beta_{n}\right)(n+1)}{2 \sqrt{n\left(n+\alpha_{n}\right)\left(n+\beta_{n}\right)\left(n+\alpha_{n}+\beta_{n}\right)}} \\
& \quad+\sqrt{\frac{(n+1)\left(\alpha_{n}+\beta_{n}+n-1\right)}{n\left(n+\alpha_{n}+\beta_{n}\right)}} \frac{w_{n}+w_{n}^{-1}}{2},
\end{aligned}
$$

$\left|w_{n}\right|>1$; that is, the exterior of the unit circle in the $w_{n}$-plane is mapped conformally onto the domain $\mathbb{C}_{\left[r_{n}, s_{n}\right]}$ in the $z$-plane such that the points $w_{n}=\infty$ and $z=\infty$ correspond to each other. Moreover, the upper and the lower edge of the cut in the $z$-plane is mapped onto the upper and lower half of the unit circle in the $w_{n}$-plane respectively. Inverting in (3.8) yields

$$
\begin{aligned}
w_{n}= & \frac{\sqrt{z-r_{n}}+\sqrt{z-s_{n}}}{\sqrt{z-r_{n}}-\sqrt{z-s_{n}}}=\frac{2}{s_{n}-r_{n}}\left(z-\frac{r_{n}+s_{n}}{2}+\sqrt{\left(z-r_{n}\right)\left(z-s_{n}\right)}\right) \\
= & \sqrt{\frac{n\left(n+\alpha_{n}+\beta_{n}\right)}{(n+1)\left(n+\alpha_{n}+\beta_{n}-1\right)}} \\
& \times\left(z-\frac{\left(\alpha_{n}-\beta_{n}\right)(n+1)}{2 \sqrt{n\left(n+\alpha_{n}\right)\left(n+\beta_{n}\right)\left(n+\alpha_{n}+\beta_{n}\right)}}+\sqrt{\left(z-r_{n}\right)\left(z-s_{n}\right)}\right) .
\end{aligned}
$$

Now putting

$$
t_{n}:=\frac{1}{2}\left(2 n+\alpha_{n}+\beta_{n}\right) \sqrt{\frac{n+1}{n\left(n+\alpha_{n}+\beta_{n}-1\right)\left(n+\alpha_{n}+\beta_{n}\right)}} w_{n}
$$

we have

$$
t_{n}=\frac{2 n+\alpha_{n}+\beta_{n}}{2\left(n+\alpha_{n}+\beta_{n}-1\right)}\left(z-\frac{r_{n}+s_{n}}{2}+\sqrt{\left(z-r_{n}\right)\left(z-s_{n}\right)}\right)
$$


that is, in (3.2) we make the choice of the branch such that the cut $z$-plane $\mathbb{C}_{\left[r_{n}, s_{n}\right]}$ is mapped conformally onto the exterior of the circle $|t|=\rho_{n}, \rho_{n}$ being the factor of $w_{n}$ in (3.10). On the cut $\left(r_{n}, s_{n}\right)$ in the $z$-plane, in view of (3.8) and (3.10), we have

$$
\begin{aligned}
z & =\frac{r_{n}+s_{n}}{2}+\frac{s_{n}-r_{n}}{2} \cos \theta, \quad w_{n}=e^{i \theta}, \quad 0<\theta<\pi, \\
t_{n} & =\frac{1}{2}\left(2 n+\alpha_{n}+\beta_{n}\right) \sqrt{\frac{n+1}{n\left(n+\alpha_{n}+\beta_{n}-1\right)\left(n+\alpha_{n}+\beta_{n}\right)}} e^{i \theta} .
\end{aligned}
$$

Now straightforward computations lead to the following connection formulae [7, Lemma 1; 2, Lemma 2.1; 8, Lemma 3].

Lemma 3.1. If the complex variables $z, w_{n}$, and $t_{n}$ are related by (3.8)-(3.11), then we have

$$
\begin{aligned}
t_{n}-z=\frac{n+1}{2 w_{n}} \sqrt{\frac{n+1}{n\left(n+\alpha_{n}+\beta_{n}-1\right)\left(n+\alpha_{n}+\beta_{n}\right)}} \\
\times\left(w_{n}-\sqrt{\frac{\left(n+\alpha_{n}+\beta_{n}-1\right)\left(n+\alpha_{n}\right)}{(n+1)\left(n+\beta_{n}\right)}}\right) \\
\times\left(w_{n}+\sqrt{\frac{\left(n+\alpha_{n}+\beta_{n}-1\right)\left(n+\beta_{n}\right)}{(n+1)\left(n+\alpha_{n}\right)}}\right)
\end{aligned}
$$

(ii)

$$
\begin{aligned}
\frac{1-B_{n}+A_{n}-\sqrt{D_{n}} t_{n}}{1-B_{n}+A_{n}-\sqrt{D_{n}} z}=\frac{2 n+\alpha_{n}+\beta_{n}}{n+\alpha_{n}+\beta_{n}-1} & \times \frac{w_{n}}{w_{n}-\sqrt{\frac{(n+1)\left(n+\beta_{n}\right)}{\left(n+\alpha_{n}+\beta_{n}-1\right)\left(n+\alpha_{n}\right)}}},
\end{aligned}
$$

$$
\begin{aligned}
\frac{1+B_{n}-A_{n}+\sqrt{D_{n}} t_{n}}{1+B_{n}-A_{n}+\sqrt{D_{n}} z}=\frac{2 n+\alpha_{n}+\beta_{n}}{n+\alpha_{n}+\beta_{n}-1} & \times \frac{w_{n}}{w_{n}+\sqrt{\frac{(n+1)\left(n+\alpha_{n}\right)}{\left(n+\alpha_{n}+\beta_{n}-1\right)\left(n+\beta_{n}\right)}}}, \\
\frac{\left(B_{n}-A_{n}+\sqrt{D_{n}} t_{n}\right)^{2}-1}{2\left(t_{n}-z\right)}= & 4 \sqrt{\frac{n\left(n+\alpha_{n}+\beta_{n}\right)}{(n+1)\left(n+\alpha_{n}+\beta_{n}-1\right)}} \\
& \times \frac{\left(n+\alpha_{n}\right)\left(n+\beta_{n}\right)}{\left(2 n+\alpha_{n}+\beta_{n}\right)^{2}} w_{n} .
\end{aligned}
$$

Next, from (2.5), we compute

$$
p_{n}^{\prime \prime}(t)=-\frac{n+1}{(t-z)^{2}}+\frac{n+\alpha_{n}}{\left(t-\left(1-B_{n}+A_{n}\right) / \sqrt{D_{n}}\right)^{2}}+\frac{n+\beta_{n}}{\left(t+\left(1+B_{n}-A_{n}\right) / \sqrt{D_{n}}\right)^{2}},
$$

yielding the following identities via Lemma 3.1. 
Lemma 3.2. If the complex variables $z, w_{n}$, and $t_{n}$ are related by (3.8)-(3.11), then we have

$$
\begin{aligned}
& p_{n}^{\prime \prime}\left(t_{n}\right)=\frac{(n+1)\left(n+\alpha_{n}+\beta_{n}-1\right)}{2 n+\alpha_{n}+\beta_{n}} \frac{1-w_{n}^{2}}{w_{n}^{2}\left(t_{n}-z\right)^{2}} \\
& =\left(\frac{2\left(n+\alpha_{n}+\beta_{n}-1\right)}{n+1}\right)^{2} \frac{n\left(n+\alpha_{n}+\beta_{n}\right)}{2 n+\alpha_{n}+\beta_{n}}\left(1-w_{n}^{2}\right) \\
& \times\left(w_{n}-\sqrt{\frac{\left(n+\alpha_{n}+\beta_{n}-1\right)\left(n+\alpha_{n}\right)}{(n+1)\left(n+\beta_{n}\right)}}\right)^{-2} \\
& \times\left(w_{n}+\sqrt{\frac{\left(n+\alpha_{n}+\beta_{n}-1\right)\left(n+\beta_{n}\right)}{(n+1)\left(n+\alpha_{n}\right)}}\right)^{-2} .
\end{aligned}
$$

Now we are in a position to derive the strong asymptotics for the Jacobi polynomial in (2.4) on the cut plane $\mathbb{C}_{[r, s]}$ (see (3.5)-(3.7)). In this context, we observe that by passing to the limit $n \rightarrow \infty$ in (3.9), we get the function

$$
v=\lim _{n \rightarrow \infty} w_{n}=\frac{\sqrt{z-r}+\sqrt{z-s}}{\sqrt{z-r}-\sqrt{z-s}}=z-\frac{r+s}{2}+\sqrt{(z-r)(z-s)},
$$

mapping $\mathbb{C}_{[r, s]}$ conformally onto the exterior of the unit circle $|v|>1$.

Theorem 3.3. Suppose that the real sequences $\left(\alpha_{n}\right),\left(\beta_{n}\right)$ satisfy (1.9) with at least one of the limits $a, b$ being infinite and $\alpha_{n}, \beta_{n} \geq 1$. If the function $w_{n}$ is defined in (3.8), then for $z \in \mathbb{C}_{[r, s]}$, the Jacobi polynomial rescaled according to (2.4) satisfies

$$
\begin{aligned}
P_{n}^{\left(\alpha_{n}, \beta_{n}\right)} & \left(\sqrt{D_{n}} z+B_{n}-A_{n}\right) \\
= & \left(\frac{2 n+\alpha_{n}+\beta_{n}}{2 \pi(n+1)\left(n+\alpha_{n}+\beta_{n}-1\right)}\right)^{1 / 2}\left(\frac{\left(n+\alpha_{n}\right)\left(n+\beta_{n}\right)}{(n+1)\left(n+\alpha_{n}+\beta_{n}-1\right)}\right)^{n / 2} \\
& \times\left\{\frac{2 n+\alpha_{n}+\beta_{n}}{n+\alpha_{n}+\beta_{n}-1} \frac{w_{n}}{w_{n}-\sqrt{\frac{(n+1)\left(n+\beta_{n}\right)}{\left(n+\alpha_{n}+\beta_{n}-1\right)\left(n+\alpha_{n}\right)}}}\right\}^{\alpha_{n}} \\
& \times\left\{\frac{2 n+\alpha_{n}+\beta_{n}}{n+\alpha_{n}+\beta_{n}-1} \frac{w_{n}}{w_{n}+\sqrt{\frac{(n+1)\left(n+\alpha_{n}\right)}{\left(n+\alpha_{n}+\beta_{n}-1\right)\left(n+\beta_{n}\right)}}}\right\}^{\beta_{n}} \\
& \times \frac{w_{n}^{n+1}}{\left(w_{n}^{2}-1\right)^{1 / 2}}(1+o(1)), \text { as } n \rightarrow \infty .
\end{aligned}
$$

Here the branches of the fractional powers are positive when $w_{n}$ is real and greater than $s_{n}$ (see (3.4)). Moreover the o-term holds uniformly on compact subsets of $\mathbb{C}_{[r, s]}$.

We emphasize that without further growth conditions on $\alpha_{n}, \beta_{n}$ in (1.9), we may not replace $w_{n}$ by $v$ in (3.14).

Proof. We consider the case $a=\infty, b \leq \infty$ in (1.9) which corresponds to the limiting cases (3.5) and (3.7) for the boundary points of the zero interval. Further, suppose that $z=x>2$. Then on account of Lemma 3.1(i), we have $t_{n}<x$ at least for sufficiently large $n$. Following arguments in $[2,7,29]$, first we establish the asymptotic formula (3.14) for real $x$ specified above, and then we transfer its validity to the cut plane $\mathbb{C}_{[r, s]}$ via Vitali's theorem. 
We introduce the quantities $\omega_{n}:=-i n^{-\delta}, 0<\delta<1 / 2$,

$$
R_{n}:=\frac{\left|x-t_{n}-\omega_{n}\right|}{x-t_{n}}
$$

and $\psi_{n} \in(0, \pi)$ according to $\pi-\psi_{n}=\arccos \left(1 / R_{n}\right)(0<\arccos \xi<\pi$ for $-1<$ $\xi<1)$. Similar to the Laguerre case in [2, Proof of Theorem 2.3], we start from the representation formula (2.4) and use $t_{n}$ as the saddle point given by (3.10). As the contour in (2.4), we choose

$$
C_{n}:=C_{n}^{u} \cup T_{n} \cup C_{n}^{l}
$$

consisting of the straight line

$$
T_{n}:=\left\{t_{n}+\tau \omega_{n} \mid-1 \leq \tau \leq 1\right\}
$$

and the circular arcs

$$
\begin{gathered}
C_{n}^{u}:=\left\{t=x+\left|x-t_{n}-\omega_{n}\right| e^{i \psi} \mid 0 \leq \psi \leq \psi_{n}\right\}, \\
C_{n}^{l}:=\left\{t=x+\left|x-t_{n}-\omega_{n}\right| e^{i \psi} \mid 2 \pi-\psi_{n} \leq \psi \leq 2 \pi\right\} .
\end{gathered}
$$

The contribution of $T_{n}$ to the integral in (2.4) is computed by Lemma 2.1. Conditions (2.6)-(2.9) are easily verified with the aid of Lemma 3.2. In particular, we have to make the choice $\arg p_{n}^{\prime \prime}\left(t_{n}\right)=\pi$. Thus we get

$$
\int_{T_{n}} e^{-p_{n}(t)} d t=\frac{1}{i} \sqrt{\frac{2 \pi}{\left|p_{n}^{\prime \prime}\left(t_{n}\right)\right|}} e^{-p_{n}\left(t_{n}\right)}(1+o(1)), \quad n \rightarrow \infty .
$$

Now the crucial point is to prove that the contributions along $C_{n}^{u}, C_{n}^{l}$ in (2.4) are negligible. We restrict our reasoning to $C_{n}^{u}$. That for $C_{n}^{l}$ is very similar. Let $t \in C_{n}^{u}$; then we estimate $\operatorname{Re}\left(p_{n}(t)-p_{n}\left(t_{n}\right)\right)$ from below. On account of (2.5) and Lemma 3.1 obvious calculations lead to

$$
\begin{aligned}
\operatorname{Re}\left(p_{n}(t)-p_{n}\left(t_{n}\right)\right)=( & n+1) \operatorname{Re} \log \frac{t-x}{t_{n}-x} \\
& -\left(n+\alpha_{n}\right) \operatorname{Re} \log \frac{\sqrt{D_{n}} t-1+B_{n}-A_{n}}{\sqrt{D_{n}} t_{n}-1+B_{n}-A_{n}} \\
& -\left(n+\beta_{n}\right) \operatorname{Re} \log \frac{\sqrt{D_{n}} t+1+B_{n}-A_{n}}{\sqrt{D_{n}} t_{n}+1+B_{n}-A_{n}} .
\end{aligned}
$$

Further, since

$$
\begin{aligned}
& \frac{1-B_{n}+A_{n}}{\sqrt{D_{n}}}=\frac{2 n+\alpha_{n}+\beta_{n}}{2} \sqrt{\frac{n+\alpha_{n}}{n\left(n+\beta_{n}\right)\left(n+\alpha_{n}+\beta_{n}\right)}} \\
& \frac{1-B_{n}+A_{n}}{\sqrt{D_{n}}}=\frac{2 n+\alpha_{n}+\beta_{n}}{2} \sqrt{\frac{n+\beta_{n}}{n\left(n+\alpha_{n}\right)\left(n+\alpha_{n}+\beta_{n}\right)}},
\end{aligned}
$$


we get

$$
\begin{aligned}
& \operatorname{Re}\left(p(t)-p_{n}\left(t_{n}\right)\right)=(n+1) \log R_{n} \\
& -\left(n+\alpha_{n}\right) \operatorname{Re} \log \left\{1-\frac{1+R_{n} e^{i \psi}}{w_{n}} \frac{n+1}{2 n+\alpha_{n}+\beta_{n}}\right. \\
& \left.\times\left(w_{n}+\sqrt{\frac{\left(n+\alpha_{n}+\beta_{n}-1\right)\left(n+\beta_{n}\right)}{(n+1)\left(n+\alpha_{n}\right)}}\right)\right\} \\
& -\left(n+\beta_{n}\right) \operatorname{Re} \log \left\{1-\frac{1+R_{n} e^{i \psi}}{w_{n}} \frac{n+1}{2 n+\alpha_{n}+\beta_{n}}\right. \\
& \left.\times\left(w_{n}-\sqrt{\frac{\left(n+\alpha_{n}+\beta_{n}-1\right)\left(n+\alpha_{n}\right)}{(n+1)\left(n+\beta_{n}\right)}}\right)\right\}
\end{aligned}
$$

for $0 \leq \psi \leq \psi_{n}$. In view of the well-known inequality,

$$
\operatorname{Re}(\zeta-\log (1+\zeta)) \geq-\frac{1}{2}|\zeta|^{2}, \quad \zeta \in \mathbb{C} \backslash\{-1\}
$$

this gives

$$
\begin{aligned}
\operatorname{Re}\left(p_{n}(t)-p_{n}(t)\right) \geq( & (n+1)\left(\log R_{n}+1+R_{n} \cos \psi\right) \\
& \quad-\frac{1}{2} \frac{(n+1)^{2}}{2 n+\alpha_{n}+\beta_{n}} \frac{\left|1+R_{n} e^{i \psi}\right|^{2}}{w_{n}^{2}}\left(w_{n}^{2}+\frac{n+\alpha_{n}+\beta_{n}-1}{n+1}\right) \\
\geq( & (n+1)\left\{\log R_{n}+1+R_{n} \cos \psi\left(1-\frac{1}{w_{n}^{2}}\right)\left(1-\frac{n+1}{2 n+\alpha_{n}+\beta_{n}}\right)\right. \\
& \left.\quad-\frac{1}{2} \frac{1+R_{n}^{2}}{w_{n}^{2}}\left(1+\frac{n+1}{2 n+\alpha_{n}+\beta_{n}}\left(w_{n}^{2}-1\right)\right)\right\}
\end{aligned}
$$

for $0 \leq \psi \leq \psi_{n}$. Using the expansion (see (3.15))

$$
R_{n}=1+\frac{1}{2} \frac{n^{-2 \delta}}{\left(x-t_{n}\right)^{2}}+O\left(n^{-4 \delta}\right), \quad n \rightarrow \infty,
$$

and observing that $R_{n} \cos \psi_{n}=-1$, finally we end with

$$
\begin{aligned}
\operatorname{Re} & \left(p_{n}(t)-p_{n}\left(t_{n}\right)\right) \\
& \geq(n+1)\left\{\frac{n^{-2 \delta}}{2\left(x-t_{n}\right)^{2}}\left(1-\frac{1}{w_{n}^{2}}\right)\left(1-\frac{n+1}{2 n+\alpha_{n}+\beta_{n}}\right)+O\left(n^{-4 \delta}\right)\right\} \\
& \geq c n^{1-2 \delta}
\end{aligned}
$$

for some positive constant $c$ and sufficiently large $n$. Lemma 3.2 shows that $\left|p_{n}^{\prime \prime}\left(t_{n}\right)\right|$ grows like a positive multiple of $n$ and so

$$
\int_{C_{n}^{u}} e^{-p_{n}(t)} d t=o\left(\int_{T_{n}} e^{-p_{n}(t)} d t\right), \quad n \rightarrow \infty .
$$

This estimate also can be verified for the contribution along $C_{n}^{l}$. Thus combining (2.4), (2.5), Lemma 3.1, and (3.16), we have proved (3.14) provided that $x>2$ and $a=\infty, b \leq \infty$. The remaining case, $a<\infty, b=\infty$, can be treated by a slight 
modification of the preceding arguments, i.e., now $C_{n}$ consists of the same straight line $T_{n}$ and circular arcs being located to the left of $T_{n}$.

Next, we transfer the validity of (3.14) to the cut plane $\mathbb{C}_{[r, s]}$. To this end, let $z \in \mathcal{C}_{\left[r_{n}, s_{n}\right]}$ and $\left|w_{n}\right|>1$, related as above. Further, we put $\zeta=r e^{i \theta}=1 / w_{n}$, and, using (1.1) similar to $[2,7,29]$, we get

$$
\begin{aligned}
& \frac{2^{\alpha_{n}+\beta_{n}+1}}{2 n+\alpha_{n}+\beta_{n}+1} \frac{\Gamma\left(n+\alpha_{n}+1\right) \Gamma\left(n+\beta_{n}+1\right)}{n ! \Gamma\left(n+\alpha_{n}+\beta_{n}+1\right)} \\
& =\int_{-1}^{1}\left|P_{n}^{\left(\alpha_{n}, \beta_{n}\right)}(x)\right|^{2}(1-x)^{\alpha_{n}}(1+x)^{\beta_{n}} d x \\
& =\sqrt{D_{n}} \int_{\left(-1+A_{n}-B_{n}\right) / \sqrt{D_{n}}}^{\left(1+A_{n}-B_{n}\right) / \sqrt{D_{n}}}\left|P^{\left(\alpha_{n}, \beta_{n}\right)}\left(\sqrt{D_{n}} t+B_{n}-A_{n}\right)\right|^{2} \\
& \quad \times\left(1-B_{n}+A_{n}-\sqrt{D_{n}} t\right)^{\alpha_{n}}\left(1+B_{n}-A_{n}+\sqrt{D_{n}} t\right)^{\beta_{n}} d t .
\end{aligned}
$$

On account of (1.5), (1.10), (3.3), and (3.4), the inclusion

$$
\left[r_{n}, s_{n}\right] \subset\left[\left(-1+A_{n}-B_{n}\right) / \sqrt{D_{n}},\left(1+A_{n}-B_{n}\right) / \sqrt{D_{n}}\right], \quad n \in \mathbb{N},
$$

is readily verified. Thus, it follows that

$$
\begin{aligned}
& \frac{2^{\alpha_{n}+\beta_{n}+1}}{2 n+\alpha_{n}+\beta_{n}+1} \frac{\Gamma\left(n+\alpha_{n}+1\right) \Gamma\left(n+\beta_{n}+1\right)}{n ! \Gamma\left(n+\alpha_{n}+\beta_{n}+1\right)} \geq \sqrt{D_{n}} \int_{r_{n}}^{s_{n}} \cdots d t \\
& =\frac{\sqrt{D_{n}}}{2} \frac{s_{n}-r_{n}}{2} \int_{-\pi}^{\pi}\left|P_{n}^{\left(\alpha_{n}, \beta_{n}\right)}\left(\sqrt{D_{n}}\left(\frac{r_{n}+s_{n}}{2}+\frac{s_{n}-r_{n}}{2} \cos \theta\right)+B_{n}-A_{n}\right)\right|^{2} \\
& \quad \times\left(1-B_{n}+A_{n}-\sqrt{D_{n}}\left(\frac{r_{n}+s_{n}}{2}+\frac{s_{n}-r_{n}}{2} \cos \theta\right)\right)^{\alpha_{n}} \\
& \quad \times\left(1+B_{n}-A_{n}+\sqrt{D_{n}}\left(\frac{r_{n}+s_{n}}{2}+\frac{s_{n}-r_{n}}{2} \cos \theta\right)\right)^{\beta_{n}}|\sin \theta| d \theta \\
& =\quad \sqrt{D_{n}} \frac{s_{n}-r_{n}}{2} \lim _{r \rightarrow 1-} \frac{1}{2} \int_{-\pi}^{\pi} \mid P_{n}^{\left(\alpha_{n}, \beta_{n}\right)}\left(\sqrt{D_{n}}\left(\frac{r_{n}+s_{n}}{2}+\frac{s_{n}-r_{n}}{2} \frac{\zeta+\zeta^{-1}}{2}\right)\right. \\
& \geq \quad \sqrt{D_{n}} \frac{s_{n}-r_{n}}{2} \pi\left(1-|\zeta|^{2}\right) \mid P_{n}^{\left(\alpha_{n}, \beta_{n}\right)}\left(\sqrt{D_{n}}\left(\frac{r_{n}+s_{n}}{2}+\frac{s_{n}-r_{n}}{2} \frac{\zeta+\zeta^{-1}}{2}\right)\right. \\
& \left.\quad+B_{n}-A_{n}\right)\left.\right|^{2}\left|\zeta^{n}\right|^{2}\left|D_{n}(\zeta)\right|^{2}
\end{aligned}
$$

where

$$
\begin{aligned}
D_{n}(\zeta) & =\exp \left\{\frac { 1 } { 4 \pi } \int _ { - \pi } ^ { \pi } \operatorname { l o g } \left[\left(1-B_{n}+A_{n}-\sqrt{D_{n}}\left(\frac{r_{n}+s_{n}}{2}+\frac{s_{n}-r_{n}}{2} \cos \theta\right)\right)^{\alpha_{n}}\right.\right. \\
& \left.\left.\times\left(1+B_{n}-A_{n}+\sqrt{D_{n}}\left(\frac{r_{n}+s_{n}}{2}+\frac{s_{n}-r_{n}}{2} \cos \theta\right)\right)^{\beta_{n}}|\sin \theta|\right] \frac{1+\zeta e^{-i \theta}}{1-\zeta e^{-i \theta}} d \theta\right\} .
\end{aligned}
$$


By Lemma 2.2 (for applications of part (i) use of the assumption $\alpha_{n}, \beta_{n} \geq 1$ ), direct computations lead to

$$
\begin{aligned}
D_{n}(\zeta)= & \frac{\sqrt{w_{n}^{2}-1}}{\sqrt{2} w_{n}}\left(\frac{\sqrt{2\left(n+\alpha_{n}\right)\left(n+\alpha_{n}+\beta_{n}-1\right)}}{2 n+\alpha_{n}+\beta_{n}}\right)^{\alpha_{n}} \\
& \times\left(1-\sqrt{\frac{\left(n+\beta_{n}\right)(n+1)}{\left(n+\alpha_{n}\right)\left(n+\alpha_{n}+\beta_{n}-1\right)}} \frac{1}{w_{n}}\right)^{\alpha_{n}} \\
& \times\left(\frac{\sqrt{2\left(n+\beta_{n}\right)\left(n+\alpha_{n}+\beta_{n}-1\right)}}{2 n+\alpha_{n}+\beta_{n}}\right)^{\beta_{n}} \\
& \times\left(1+\sqrt{\frac{\left(n+\alpha_{n}\right)(n+1)}{\left(n+\beta_{n}\right)\left(n+\alpha_{n}+\beta_{n}-1\right)}} \frac{1}{w_{n}}\right)^{\beta_{n}} .
\end{aligned}
$$

Hence, for $z \in \mathbb{C}_{\left[r_{n}, s_{n}\right]}$, we get

$$
\begin{aligned}
& \mid P_{n}^{\left(\alpha_{n}, \beta_{n}\right)}\left(\sqrt{D_{n}} z+B_{n}-A_{n}\right)\left(\frac{2 \pi(n+1)\left(n+\alpha_{n}+\beta_{n}-1\right)}{2 n+\alpha_{n}+\beta_{n}}\right)^{1 / 2} \\
& \quad \times\left(\frac{(n+1)\left(n+\alpha_{n}+\beta_{n}-1\right)}{\left(n+\alpha_{n}\right)\left(n+\beta_{n}\right)}\right)^{n / 2} \\
& \quad \times\left(\frac{n+\alpha_{n}+\beta_{n}-1}{2 n+\alpha_{n}+\beta_{n}} \frac{w_{n}-\sqrt{\frac{(n+1)\left(n+\beta_{n}\right)}{\left(n+\alpha_{n}+\beta_{n}-1\right)\left(n+\alpha_{n}\right)}}}{w_{n}}\right)^{\alpha_{n}} \\
& \quad \times\left(\frac{n+\alpha_{n}+\beta_{n}-1}{2 n+\alpha_{n}+\beta_{n}} \frac{w_{n}+\sqrt{\frac{(n+1)\left(n+\alpha_{n}\right)}{\left(n+\alpha_{n}+\beta_{n}-1\right)\left(n+\beta_{n}\right)}}}{w_{n}}\right)^{\beta_{n}} \frac{\sqrt{w_{n}^{2}-1}}{w_{n}^{n+1}} \mid \\
& \leq \frac{\sqrt{2}\left|w_{n}\right|}{\sqrt{\left|w_{n}\right|^{2}-1}} K_{n} \rightarrow \frac{\sqrt{2}|v|}{\sqrt{|v|^{2}-1}},
\end{aligned}
$$

by (3.13) and Stirling's formula. Consequently, the left-hand side of (3.17) is uniformly bounded on compact subsets of $\mathbb{C}_{[r, s]}$, and thus Vitali's theorem on compact convergence for sequences of analytic functions implies the full statement of the theorem.

As a consequence of the strong asymptotics in Theorem 3.3, we obtain the weak asymptotics for the rescaled Jacobi polynomials $P_{n}^{\left(\alpha_{n}, \beta_{n}\right)}\left(\sqrt{D_{n}} z+B_{n}-A_{n}\right)$ by forming the limit

$$
\lim _{n \rightarrow \infty} \frac{1}{n} \frac{d}{d z} \log P_{n}^{\left(\alpha_{n}, \beta_{n}\right)}\left(\sqrt{D_{n}} z+B_{n}-A_{n}\right)=\frac{2}{z+\frac{r+s}{2}+\sqrt{(z-r)(z-s)}},
$$

$z \in \mathbb{C}_{[r, s]}$, which is possible in view of the uniform convergence on compact subsets of $\mathbb{C}_{[r, s]}$ in (3.16). Stieljes inversion for the right-hand side of (3.18) leads to the generalized semicircle density

$$
\frac{2}{\pi} \frac{\sqrt{(s-x)(x-r)}}{1+2(r+s) x}, \quad r<x<s
$$


being the density of the limit distribution of the zeros of the rescaled Jacobi polynomials under consideration. A different derivation of (3.19) based on the differential equation for Jacobi polynomials is contained in [5, Theorem 2]. If $a=b=\infty$, then we have $r=-1, s=1$ (see (3.7)) and (3.19) reduces to the density of the classical semicircle law, which also is well known to be the weak limit of the zeros of the rescaled Hermite polynomials $H_{n}(\sqrt{2 n+1} z)$ [e.g., 4-6, 19, 23, 30]. This coincidence of the weak asymptotics gives rise to the question: How are $P_{n}^{\left(\alpha_{n}, \beta_{n}\right)}\left(\sqrt{D_{n}} z+B_{n}-A_{n}\right)$ and $H_{n}(\sqrt{2 n+1} z)$ related asymptotically when $z \in \mathbb{C}_{[-1,1]}$ ? In view of the known strong asymptotics ( $w$ is defined in (1.4))

$$
H_{n}(\sqrt{2 n+1} z)=\frac{2^{n / 2} \sqrt{n !}}{(2 \pi n)^{1 / 4}} \frac{w^{n+1}}{\left(w^{2}-1\right)^{1 / 2}} \exp \left(\left(n+\frac{1}{2}\right) \frac{w^{2}+1}{2 w^{2}}\right)(1+o(1))
$$

as $n \rightarrow \infty$, holding uniformly on compact subsets of $\mathbb{C}_{[-1,1]}$ [e.g., $\left.7,25,29\right]$, on the basis of Theorem 3.3, it is not difficult to give various answers to the above raised question. We restrict our explanations to two cases of ultraspherical polynomials which are formulated by the following relative asymptotics.

Theorem 3.4. Suppose that the function $w$ is defined in (1.4). If the real sequence $\left(\alpha_{n}\right)$ satisfies

$$
\lim _{n \rightarrow \infty} \frac{n^{2}}{\alpha_{n}}=0
$$

then

$$
\lim _{n \rightarrow \infty} \frac{n ! 2^{n}}{\alpha_{n}^{n / 2}} \frac{P_{n}^{\left(\alpha_{n}, \alpha_{n}\right)}\left(\sqrt{1-4 A_{n}^{2}} z\right)}{H_{n}(\sqrt{2 n+1} z)}=\exp \left(\frac{-3-w^{4}}{4 w^{2}\left(w^{2}-1\right)}\right)
$$

whereas

$$
\lim _{n \rightarrow \infty} \frac{n ! 2^{n}}{n^{n}} \frac{P_{n}^{\left(n^{2}, n^{2}\right)}\left(\frac{\sqrt{1+2 n}}{n+1} z\right)}{H_{n}(\sqrt{2 n+1} z)}=\exp \left(\frac{1-5 w^{2}-2 w^{4}-2 w^{6}}{8 w^{4}\left(w^{2}-1\right)}\right)
$$

and both formulae (3.21), (3.22) hold uniformly on compact subsets of $\mathbb{C}_{[-1,1]}$.

Both limit relations of this theorem follow by tedious but straightforward computations based on Theorem 3.3 and (3.20) as explained above. Therefore, we omit the detailed calculations. Finally, in this section, we give the complementary part of the generalized Darboux formula (3.14) that is the oscillatory asymptotics on the interval of zeros.

Theorem 3.5. Suppose that the real sequences $\left(\alpha_{n}\right),\left(\beta_{n}\right)$ satisfy $(1.9)$ with at least one of the limits $a, b$ being infinite. Further assume that $\theta \in(0, \pi)$ is fixed and the functions $\rho_{n}$ and $a_{n}$ are defined through 


$$
\begin{aligned}
\rho_{n}(\theta) & =n \theta+\frac{\theta}{2}+\frac{\pi}{4} \\
& +\alpha_{n}\left\{\frac{\theta}{2}-\arctan \frac{\sqrt{\left(n+\alpha_{n}\right)\left(n+\alpha_{n}+\beta_{n}-1\right)}+\sqrt{(n+1)\left(n+\beta_{n}\right)}}{\sqrt{\left(n+\alpha_{n}\right)\left(n+\alpha_{n}+\beta_{n}-1\right.}-\sqrt{(n+1)\left(n+\beta_{n}\right)}} \tan \frac{\theta}{2}\right\} \\
& +\beta_{n}\left\{\frac{\theta}{2}-\arctan \frac{\sqrt{\left(n+\beta_{n}\right)\left(n+\alpha_{n}+\beta_{n}-1\right)}-\sqrt{(n+1)\left(n+\alpha_{n}\right)}}{\sqrt{\left(n+\beta_{n}\right)\left(n+\alpha_{n}+\beta_{n}-1\right)}+\sqrt{(n+1)\left(n+\alpha_{n}\right)}} \tan \frac{\theta}{2}\right\}
\end{aligned}
$$

$$
\begin{aligned}
& a_{n}(\theta)=\left(\frac{2 n+\alpha_{n}+\beta_{n}}{\pi(n+1)\left(n+\alpha_{n}+\beta_{n}-1\right) \sin \theta}\right)^{1 / 2} \\
& \quad \times\left(\frac{\left(n+\alpha_{n}\right)\left(n+\beta_{n}\right)}{(n+1)\left(n+\alpha_{n}+\beta_{n}-1\right)}\right)^{n / 2}\left(\frac{2 n+\alpha_{n}+\beta_{n}}{n+\alpha_{n}+\beta_{n}-1}\right)^{\alpha_{n}+\beta_{n}} \\
& \quad \times\left(1-2 \cos \theta \sqrt{\frac{(n+1)\left(n+\beta_{n}\right)}{\left(n+\alpha_{n}\right)\left(n+\alpha_{n}+\beta_{n}-1\right)}}+\frac{(n+1)\left(n+\beta_{n}\right)}{\left(n+\alpha_{n}\right)\left(n+\alpha_{n}+\beta_{n}-1\right)}\right)^{-\alpha_{n} / 2} \\
& \quad \times\left(1+2 \cos \theta \sqrt{\frac{(n+1)\left(n+\alpha_{n}\right)}{\left(n+\beta_{n}\right)\left(n+\alpha_{n}+\beta_{n}-1\right)}}+\frac{(n+1)\left(n+\alpha_{n}\right)}{\left(n+\beta_{n}\right)\left(n+\alpha_{n}+\beta_{n}-1\right)}\right)^{-\beta_{n} / 2} .
\end{aligned}
$$

If

$$
x=\frac{\left(\alpha_{n}-\beta_{n}\right)(n+1)}{2 \sqrt{n\left(n+\alpha_{n}\right)\left(n+\beta_{n}\right)\left(n+\alpha_{n}+\beta_{n}\right)}}+\sqrt{\frac{(n+1)\left(n+\alpha_{n}+\beta_{n}-1\right)}{n\left(n+\alpha_{n}+\beta_{n}\right)}} \cos \theta,
$$

then the rescaled Jacobi polynomial in (2.4) satisfies

$$
P_{n}^{\left(\alpha_{n}, \beta_{n}\right)}\left(\sqrt{D_{n}} x+B_{n}-A_{n}\right)=a_{n}(\theta)\left\{\sin \rho_{n}(\theta)+o(1)\right\}
$$

as $n \rightarrow \infty$.

In (3.23), "arctan" denotes the principal branch, that is $-\frac{\pi}{2}<\arctan \xi<\frac{\pi}{2}$ for real $\xi$. Moreover, from general estimates for the remainder of the saddle point approximation $[20,31]$, it can be shown that the error term in (3.25) holds uniformly in $\theta \in[\epsilon, \pi-\epsilon]$. To a large extent, the proof of Theorem 3.5 is similar to that of Theorem 3.3 and the determination of the quantities in (3.23), (3.24) follows the calculations in the proof of Theorem 1 in [8]. Therefore, we omit the details. We only mention that the function $\rho_{n}$ in (3.23) satisfies $\rho_{n}(\theta)=\frac{n}{2}(2 \theta-\sin 2 \theta)(1+o(1)), n \rightarrow \infty$, which indicates the similarity to the corresponding Plancherel-Rotach formula in [25, p. 201 (8.22.12)] for Hermite polynomials.

\section{The case $\alpha_{n} / n \rightarrow a$ and $\beta_{n} / n \rightarrow b$}

As already mentioned in the introduction, the special case of linear parameter sequences $\alpha_{n}=a n+\alpha, \beta_{n}=b n+\beta, a, b \geq 0$, has been dealt with extensively in $[3,8]$. Now, more generally, we impose condition (1.9) with $a, b \in[0, \infty)$ both being finite. In contrast to the preceding section, here, as in the case of the classical Darboux formulae (1.2), (1.3), we consider the nonrescaled Jacobi polynomial $P_{n}^{\left(\alpha_{n}, \beta_{n}\right)}(z)$ represented by (2.2). Again we apply the saddle point approximation via Lemma 2.1. 
Since the calculations are very similar to those in Section 3, we only state the resulting two theorems below. To this end, we modify the quantities $r_{n}, s_{n}, w_{n}$ (see (3.3), (3.4), (3.9)) and introduce the notations

$$
\begin{gathered}
r_{n}^{*}=\frac{\beta_{n}\left(\beta_{n}-2\right)-\alpha_{n}\left(\alpha_{n}-2\right)-4 \sqrt{(n+1)\left(n+\alpha_{n}\right)\left(n+\beta_{n}\right)\left(n+\alpha_{n}+\beta_{n}-1\right)}}{\left(2 n+\alpha_{n}+\beta_{n}\right)^{2}}, \\
s_{n}^{*}=\frac{\beta_{n}\left(\beta_{n}-2\right)-\alpha_{n}\left(\alpha_{n}-2\right)+4 \sqrt{(n+1)\left(n+\alpha_{n}\right)\left(n+\beta_{n}\right)\left(n+\alpha_{n}+\beta_{n}-1\right)}}{\left(2 n+\alpha_{n}+\beta_{n}\right)^{2}} \\
w_{n}^{*}=\frac{2}{s_{n}^{*}-r_{n}^{*}}\left(z-\frac{r_{n}^{*}+s_{n}^{*}}{2}+\sqrt{\left(z-r_{n}^{*}\right)\left(z-s_{n}^{*}\right)}\right)
\end{gathered}
$$

the square root being chosen such that the cut $z$-plane $\mathbb{C}_{\left[r_{n}^{*}, s_{n}^{*}\right]}$ is mapped conformally onto the exterior of the unit circle $\left|w_{n}^{*}\right|>1$. Obviously, we have $r_{n}^{*} \rightarrow r, s_{n}^{*} \rightarrow s$ where $r, s$ are given by (1.7). Now we have

Theorem 4.1. Suppose that the real sequences $\left(\alpha_{n}\right),\left(\beta_{n}\right)$ satisfy $(1.9)$ with both limits $a, b$ being finite. If the function $w_{n}^{*}$ is defined in (4.3), then for $z \in \mathbb{C}_{[r, s]}$ the Jacobi polynomial $P_{n}^{\left(\alpha_{n}, \beta_{n}\right)}(z)$ satisfies

$$
\begin{aligned}
P_{n}^{\left(\alpha_{n}, \beta_{n}\right)}(z)= & \left.\frac{2 n+\alpha_{n}+\beta_{n}}{2 \pi(n+1)\left(n+\alpha_{n}+\beta_{n}-1\right)}\right)^{1 / 2}\left(\frac{\left(n+\alpha_{n}\right)\left(n+\beta_{n}\right)}{(n+1)\left(n+\alpha_{n}+\beta_{n}-1\right)}\right)^{n / 2} \\
& \times\left\{\frac{2 n+\alpha_{n}+\beta_{n}}{n+\alpha_{n}+\beta_{n}-1} \frac{w_{n}^{*}}{w_{n}^{*}-\sqrt{\frac{(n+1)\left(n+\beta_{n}\right)}{\left(n+\alpha_{n}+\beta_{n}-1\right)\left(n+\alpha_{n}\right)}}}\right\}^{\alpha_{n}} \\
& \times\left\{\frac{2 n+\alpha_{n}+\beta_{n}}{n+\alpha_{n}+\beta_{n}-1} \frac{w_{n}^{*}}{w_{n}^{*}+\sqrt{\frac{(n+1)\left(n+\alpha_{n}\right)}{\left(n+\alpha_{n}+\beta_{n}-1\right)\left(n+\beta_{n}\right)}}}\right\}^{\beta_{n}} \\
& \times \frac{w_{n}^{* n+1}}{\left(w_{n}^{* 2}-1\right)^{1 / 2}}(1+o(1)), \text { as } n \rightarrow \infty .
\end{aligned}
$$

Concerning the fractional powers and the uniform validity on $\mathbb{C}_{[r, s]}$, analogous properties hold as in Theorem 3.3.

Theorem 4.2. Suppose that the real sequences $\left(\alpha_{n}\right),\left(\beta_{n}\right)$ satisfy $(1.9)$ with both limits $a, b$ being finite. Further assume that $\theta \in(0, \pi)$ is fixed and the functions $\rho_{n}, a_{n}$ are defined in (3.23), (3.24), respectively. If

$$
x=\frac{\beta_{n}\left(\beta_{n}-2\right)-\alpha_{n}\left(\alpha_{n}-2\right)+4 \sqrt{(n+1)\left(n+\alpha_{n}\right)\left(n+\beta_{n}\right)\left(n+\alpha_{n}+\beta_{n}-1\right)} \cos \theta}{\left(2 n+\alpha_{n}+\beta_{n}\right)^{2}}
$$

then the right-hand side of (3.25) also serves as an asymptotic form for the Jacobi polynomial $P_{n}^{\left(\alpha_{n}, \beta_{n}\right)}(x)$. 
Arguing as in Section 3 from Theorem 4.1, we can derive the known limit distribution of the zeros of $P_{n}^{\left(\alpha_{n}, \beta_{n}\right)}$ under the assumptions on $\alpha_{n}, \beta_{n}$ of this section [2, 7, 8, $29]$.

\section{References}

1. L. Berg, Asymptotische Darstellungen und Entwicklungen, VEB Deutscher Verlag der Wissenschaften, Berlin, 1968.

2. C. Bosbach and W. Gawronski, Strong asymptotics for Laguerre polynomials with varying weights, J. Comput. Appl. Math., to appear.

3. Li-chen Chen and M. E. H. Ismail, On asymptotics of Jacobi polynomials, SIAM J. Math. Anal. 22 (1991), 1442-1449.

4. H. Dette and W. J. Studden, Some new asymptotic properties for the zeros of Jacobi, Laguerre, and Hermite polynomials, Constr. Approx. 11 (1995), 227-238.

5. J. Faldey and W. Gawronski, On the limit distributions of the zeros of Jonquière polynomials and generalized classical orthogonal polynomials, J. Approx. Theory 81 (1995), 231-249.

6. W. Gawronski, On the asymptotic distribution of the zeros of Hermite, Laguerre and Jonquière polynomials, J. Approx. Theory 50 (1987), 214-231.

7. W. Gawronski, Strong asymptotics and the asymptotic zero distributions of Laguerre polynomials $L_{n}^{(a n+\alpha)}$ and Hermite polynomials $H_{n}^{(a n+\alpha)}$, Analysis 13 (1993), 29-67.

8. W. Gawronski and B. L. R. Shawyer, Strong asymptotics and the limit distribution of the zeros of Jacobi polynomials $P_{n}^{(a n+\alpha, b n+\beta)}$. In: Progress in Approximation Theory (Eds. P. Nevai and A. Pinkus), Academic Press, New York, 1991, pp. 379-404.

9. A. A. Gonchar and E. A. Rakhmanov, On convergence of simultaneous Padé approximants for systems of Markov type, Proc. Steklov Inst. Math. 157 (1983), 31-50.

10. _ Equilibrium measure and the distribution of zeros of extremal polynomials, Math. USSR Sbornik 53 (1986), 119-130.

11. P. Henrici, Applied and Computational Complex Analysis, vol. II, J. Wiley, New York, 1977.

12. M. Lachance, E. B. Saff, and R. S. Varga, Bounds for incomplete polynomials vanishing at both endpoints of an interval. In: Constructive Approaches to Mathematical Models (Eds. C. V. Coffman and G. J. Fix), Academic Press, New York, 1989, pp. 421-437.

13. D. S. Lubinsky, A survey of general orthogonal polynomials for weights on finite and infinite intervals, Acta Appl. Math. 10 (1987), 237-296.

14. __ Strong Asymptotics for Extremal Errors and Polynomials Associated with Erdös-Type Weights, Longman, Harlow, Essex, UK, 1989.

15. D. S. Lubinsky and E. B. Saff, Strong Asymptotics for Extremal Polynomials Associated with Weights on $\mathbb{R}$, Springer, Berlin, 1988.

16. H. N. Mhaskar and E. B. Saff, Weighted polynomials on finite and infinite intervals: a unified approach, Bull. Amer. Math. Soc. 11 (1984), 351-354.

17. polynomials), Constr. Approx. 1 (1985), 71-91.

18. D. Moak, E. B. Saff, and R. S. Varga, On the zeros of Jacobi polynomials $P_{n}^{\left(\alpha_{n}, \beta_{n}\right)}(x)$, Trans. Amer. Math. Soc. 249 (1979), 159-162.

19. P. Nevai, Orthogonal Polynomials, Amer. Math. Soc., Providence, RI, 1979.

20. F. Olver, Introduction to Asymptotics and Special Functions, Academic Press, New York, 1974.

21. E. A. Rakhmanov, Strong asymptotics for orthogonal polynomials. In: Methods of Approximation Theory in Complex Analysis and Mathematical Physics, Springer, Berlin, 1993, pp. 71-97.

22. E. B. Saff and R. S. Varga, The sharpness of Lorentz's theorem on incomplete polynomials, Trans. Amer. Math. Soc. 249 (1979), 163-186.

23. E. B. Saff, J. L. Ullman, and R. S. Varga, Incomplete polynomials: An electrostatics approach. In: Approximation Theory II (Ed. E. W. Cheney), Academic Press, New York, 1980, pp. 769-782.

24. E. B. Saff and V. Totik, Logarithmic Potentials with External Fields, Springer, Berlin, 1997.

25. G. Szegö, Orthogonal Polynomials, Amer. Math. Soc., Providence, RI, 1975.

26. N. Temme, Polynomial asymptotic estimates of Gegenbauer, Laguerre and Jacobi polynomials. In: Asymptotic and Computational Analysis (Ed. R. Wong), Marcel Dekker, New York, 1990, pp. $455-476$. 
27. N. Temme, Special Functions, J. Wiley, New York, 1996.

28. V. Totik, Weighted Approximation with Varying Weight, Springer, Berlin, 1994.

29. W. Van Assche, Some recent results on the asymptotic distribution of the zeros of orthogonal polynomials, J. Comput. Appl. Math. 12 \& 13 (1985), 615-623.

30. W. Van Assche, Asymptotics for Orthogonal Polynomials, Springer, Berlin, 1987.

31. R. Wong, Asymptotic Approximations of Integrals, Academic Press, New York, 1989.

Abteilung Mathematik, Universität Trier, D-54286 Trier, Germany

E-mail: gawron@uni-trier.de 